\title{
Robot-assisted carotid artery stenting: outcomes, safety, and operational learning curve
}

\author{
Rawad Abbas, MD, ${ }^{1}$ Fadi AI Saiegh, MD, ${ }^{1}$ Kareem El Naamani, MD, ${ }^{1}$ Ching-Jen Chen, MD, ${ }^{1}$ \\ Lohit Velagapudi, BS, ${ }^{1}$ Georgios S. Sioutas, MD, ${ }^{1}$ Joshua H. Weinberg, MD, ${ }^{2}$ \\ Stavropoula Tjoumakaris, MD, ${ }^{1}$ M. Reid Gooch, MD, ${ }^{1}$ Nabeel A. Herial, MD, MPH, ${ }^{1}$ \\ Robert H. Rosenwasser, MD, MBA, ${ }^{1}$ and Pascal Jabbour, MD ${ }^{1}$
}

\begin{abstract}
${ }^{1}$ Department of Neurological Surgery, Thomas Jefferson University Hospital, Philadelphia, Pennsylvania; and 2Department of Neurological Surgery, The Ohio State University Wexner Medical Center, Columbus, Ohio
\end{abstract}

\begin{abstract}
OBJECTIVE Over the past 2 decades, robots have been increasingly used in surgeries to help overcome human limitations and perform precise and accurate tasks. Endovascular robots were pioneered in interventional cardiology, however, the CorPath GRX was recently approved by the FDA for peripheral vascular and extracranial interventions. The authors aimed to evaluate the operational learning curve for robot-assisted carotid artery stenting over a period of 19 months at a single institution.
\end{abstract}

METHODS A retrospective analysis of a prospectively maintained database was conducted, and 14 consecutive patients who underwent robot-assisted carotid artery stenting from December 2019 to June 2021 were identified. The metrics for proficiency were the total fluoroscopy and procedure times, contrast volume used, and radiation dose. To evaluate operator progress, the patients were divided into 3 groups of 5,4 , and 5 patients based on the study period.

RESULTS A total of 14 patients were included. All patients received balloon angioplasty and stent placement. The median degree of stenosis was $95 \%$. Ten patients (71\%) were treated via the transradial approach and 4 patients $(29 \%)$ via the transfemoral approach, with no procedural complications. The median contrast volume used was $80 \mathrm{~mL}$, and the median radiation dose was $38,978.5 \mathrm{mGy} / \mathrm{cm}^{2}$. The overall median fluoroscopy and procedure times were 24.6 minutes and 70.5 minutes, respectively. Subgroup analysis showed a significant decrease in these times, from 32 minutes and 86 minutes, respectively, in group 1 to 21.9 minutes and 62 minutes, respectively, in group $3(p=0.002$ and $p=0.008$, respectively).

CONCLUSIONS Robot-assisted carotid artery stenting was found to be safe and effective, and the learning curve for robotic procedures was overcome within a short period of time at a high-volume cerebrovascular center.

https://thejns.org/doi/abs/10.3171/2021.10.FOCUS21504

KEYWORDS angioplasty; carotid stenosis; Corindus; endovascular; robot; robot-assisted; stent

$\mathrm{T}$ ECHNOLOGY in surgery is intended to optimize patient outcomes while minimizing complications and adverse events. Specifically, advancement in robotic engineering may aid operators in repetitive, challenging tasks with pinpoint precision and accuracy, ${ }^{1}$ thereby overcoming the limits of physical ability and inherent human error, as well as limiting occupational hazards such as radiation exposure and infectious disease., ${ }^{1,2}$ Additionally, a great merit of robotic surgeries is the ability to perform them at remote locations, which would essentially provide lifesaving procedures to patients who are unable to present to a cerebrovascular center in a timely fashion. ${ }^{2-6}$ Historically, robots were largely designed for other surgical specialties, mainly interventional cardiology; ${ }^{1}$ it was more recently that they were engineered to assist in neurosurgical procedures such as depth electrode implantation for epilepsy, and minimally invasive spine surgeries. ${ }^{7,8}$

The CorPath GRX (Corindus Inc.) robot-assisted platform is the latest robotic system initially designed for interventional cardiology procedures. However, based on the results of prior clinical studies on its predecessor, the CorPath 200 (Corindus Inc.), ${ }^{9,10}$ the robotic platform was approved by the US FDA and received a CE mark for percutaneous coronary (2016) and peripheral vascular (2018) interventions. ${ }^{1}$ Following approval, a few studies have reported on the early experience with the CorPath GRX with

ABBREVIATIONS $\mathrm{mRS}=$ modified Rankin Scale; $\mathrm{TIA}=$ transient ischemic attack. 

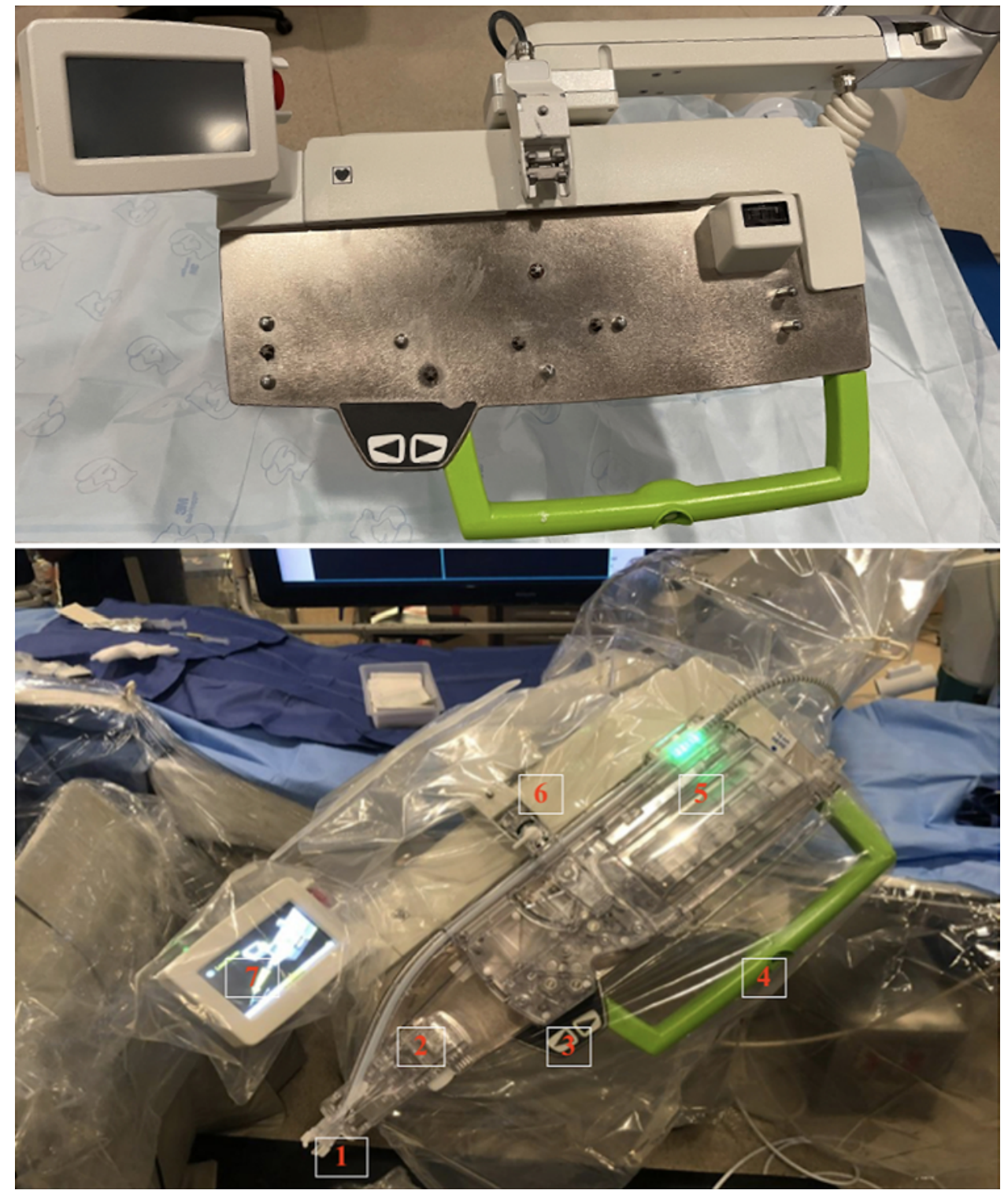

FIG. 1. Upper: Robotic arm that holds the disposable cassette. Lower: Disposable cassette (1 use per procedure). $1=$ sheath attachment; 2 = guide catheter rotation module; 3 = microadjustment buttons; 4 = robotic arm toggle button; 5 = guidewire rotation module; 6 = cassette lock; 7 = robotic arm feedback console.

neuroendovascular procedures such as diagnostic cerebral angiograms, carotid artery stenting, and a stent-assisted coil embolization of a basilar artery aneurysm.,2,11,12 The latter was performed in Canada, as the CorPath robot is not yet approved for intracranial procedures in the US. In the present study, we looked at the outcome, safety, and learning curve for robot-assisted carotid artery stenting over a period of 19 months.

\section{Methods \\ Study Population}

In this retrospective study, the data of 14 patients, who were treated from December 2019 to June 2021, were collected from a single tertiary care, high-volume cerebrovascular center in the US. All patients treated with robot-assisted carotid artery stenting were included. The CorPath GRX robot-assisted platform was used for the procedures (Figs. 1 and 2). Three surgeons were involved with the procedures, as follows. The first surgeon had 11 cases; the second, 2 cases; and the third, 1 case. The study protocol was reviewed and approved by the IRB. Patient consent was not obtained due to the retrospective nature of the study. The following data were collected from each patient's electronic medical record, from a prospectively maintained database: age, sex, history of hypertension, history of diabetes mellitus, history of coronary artery disease, history of hyperlipidemia, presentation (stroke, transient ischemic attack [TIA], or asymptomatic), smoking status, baseline modified Rankin Scale (mRS) score, medication information (antiplatelet, anticoagulation, and 
Abbas et al.

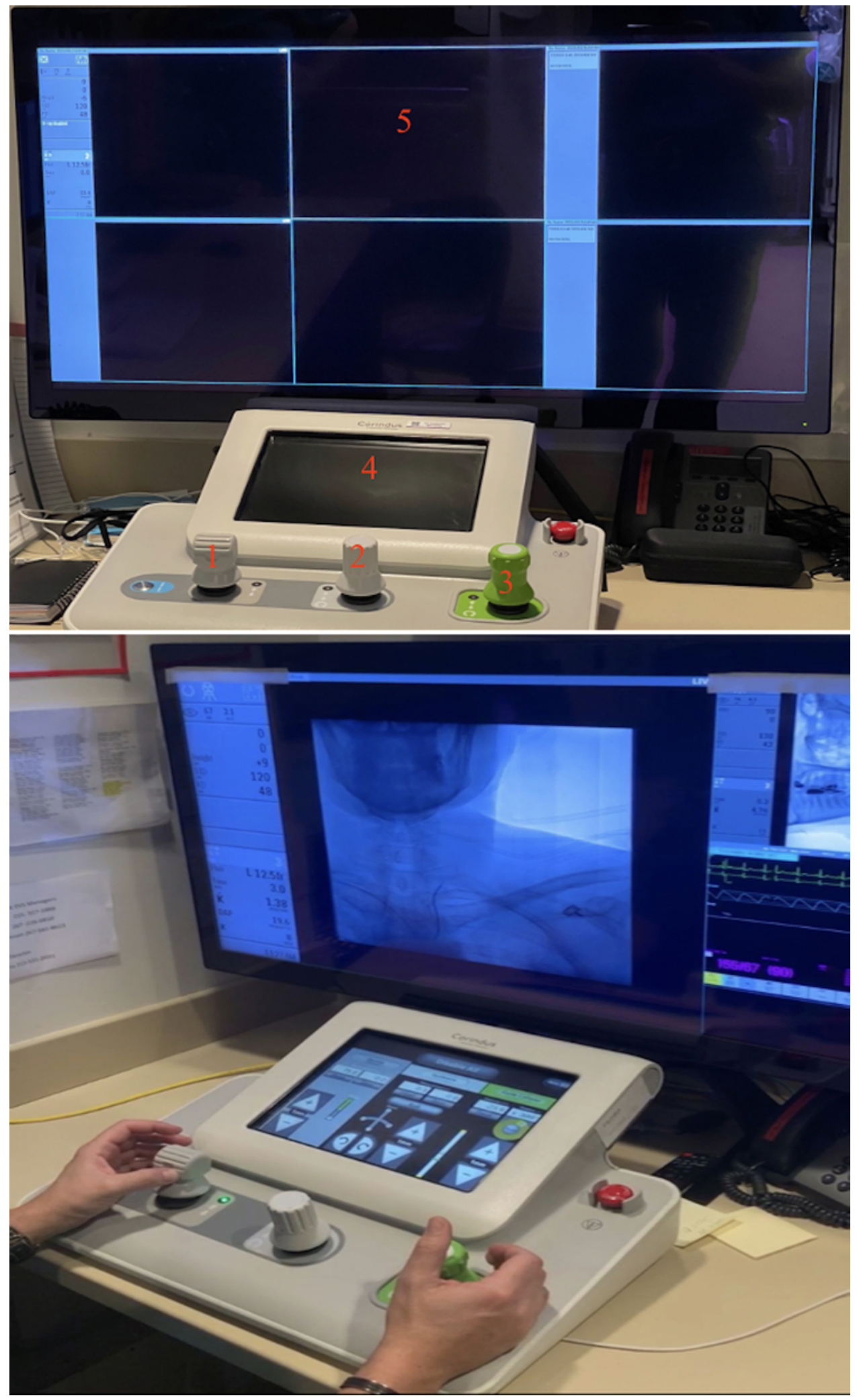

FIG. 2. Upper: Remote physician unit. 1 = device joystick; 2 = guidewire joystick; 3 = catheter joystick; 4 = joystick feedback monitor; 5 = high-definition screen. Lower: Physician maneuvering the joysticks during a procedure. 
TABLE 1. Demographics and baseline characteristics

\begin{tabular}{|c|c|c|c|c|c|c|c|c|c|c|}
\hline \multirow[b]{2}{*}{ Pt No. } & \multicolumn{10}{|c|}{ Variable } \\
\hline & Age (yrs) & F Sex & DM & HTN & CAD & TIA & Stroke & Asymptomatic & Baseline mRS Score 0 or 1 & Current Smoker \\
\hline 1 & 75 & No & Yes & No & No & No & Yes & No & Yes & No \\
\hline 2 & 77 & No & No & No & No & Yes & No & Yes & Yes & No \\
\hline 3 & 74 & No & No & Yes & No & No & Yes & No & Yes & No \\
\hline 4 & 60 & No & No & Yes & No & No & Yes & No & Yes & Yes \\
\hline 5 & 72 & No & Yes & Yes & Yes & Yes & No & No & Yes & No \\
\hline 6 & 62 & Yes & No & Yes & Yes & No & Yes & No & Yes & Yes \\
\hline 7 & 78 & No & No & Yes & No & No & No & Yes & Yes & No \\
\hline 8 & 65 & No & No & Yes & Yes & No & Yes & No & Yes & Yes \\
\hline 9 & 51 & No & Yes & Yes & Yes & Yes & No & No & Yes & No \\
\hline 10 & 84 & Yes & No & Yes & Yes & No & No & No & Yes & No \\
\hline 11 & 71 & No & No & Yes & Yes & No & Yes & No & Yes & Yes \\
\hline 12 & 55 & No & No & No & No & No & Yes & No & Yes & Yes \\
\hline 13 & 73 & Yes & No & Yes & No & No & No & Yes & Yes & No \\
\hline 14 & 77 & No & Yes & Yes & No & No & Yes & No & Yes & No \\
\hline Total no. (\%) & $72.5^{\star}$ & $3(21.4)$ & $4(28.6)$ & $11(78.6)$ & $6(42.9)$ & $3(21.4)$ & $8(57.1)$ & $3(21.4)$ & $14(100)$ & $5(35.7)$ \\
\hline
\end{tabular}

$\mathrm{CAD}=$ coronary artery disease; $\mathrm{DM}=$ diabetes mellitus; $\mathrm{HTN}=$ hypertension; $\mathrm{pt}=$ patient.

* Presented as the mean age.

statin), procedural data, complication data, hospital length of stay, and follow-up data for functional outcomes. To preserve patient anonymity, and in compliance with IRB regulations, we do not present the full data for individual cases and instead report the aggregated data or individual deidentified data points.

\section{Statistical Analysis}

All statistical analyses were performed using Stata version 17.0 (StataCorp LLC) software. Descriptive statistics were summarized using median and IQR for continuous variables and proportions for categorical variables. To analyze procedural performance measures cumulatively, the procedures were sorted by the order in which they were performed over the study period. They were then divided into 3 groups of 5,4 , and 5 patients, from the earliest to the last procedure performed. Tests for performance measure trends, including total contrast volume used (milliliters), fluoroscopy time (minutes), total procedure time (minutes), and radiation dose $\left(\mathrm{mGy} / \mathrm{cm}^{2}\right)$, were performed across groups using the Jonckheere-Terpstra test. Due to the small sample size of the study, exact $p$ values were calculated from Monte Carlo permutation tests of 100,000 random permutations. Missing data were not imputed. All tests were two-tailed, and a $\mathrm{p}$ value of $<0.05$ was considered statistically significant.

\section{Results}

\section{Demographics and Baseline Characteristics}

Overall, 14 patients were treated with a robot-assisted carotid stent procedure at our institution, and the decision to use the robot was at the discretion of the surgeon. Three patients (21\%) were female and $11(79 \%)$ were male. The mean age of the cohort was 72.5 years (range 60-84 years). The most common comorbidities were hypertension $(79 \%)$, coronary artery disease $(43 \%)$, and diabetes mellitus (29\%). Additionally, 8 patients $(57 \%)$ presented with acute ischemic stroke, 3 patients $(21 \%)$ presented with a TIA, and 3 patients $(21 \%)$ were asymptomatic. Moreover, 5 patients (36\%) were current smokers, and 3 patients $(21 \%)$ were former smokers. Five patients $(36 \%)$ were on a regimen of dual antiplatelet therapy (4 were receiving aspirin and clopidogrel, and 1 was receiving aspirin and ticagrelor) while 7 patients (50\%) were on a single antiplatelet regimen (6 were receiving aspirin and 1 was receiving clopidogrel). All patients (100\%) had an mRS score of 0 or 1 at baseline. Patient characteristics are summarized in Table 1.

\section{Procedural Details and Robotic Parameters}

Of the 14 patients, $10(71 \%)$ were treated via the transradial approach and 4 (29\%) via the transfemoral. All patients (100\%) received a Precise Pro stent (Cordis, Cardinal Health) and underwent angioplasty with the Aviator (3 patients) or Aviator Plus (11 patients) balloon. Moreover, the median degree of stenosis (based on the North American Symptomatic Carotid Endarterectomy Trial [NASCET] criteria) was 95\% (IQR 80\%-95\%), and the median stenosis diameter was $1.45 \mathrm{~mm}$ (IQR $0.8-1.8 \mathrm{~mm}$ ) (Table 2).

We used 4 objective metrics to determine the proficiency of robot-assisted carotid artery stenting: contrast volume, fluoroscopy time, procedure time, and radiation dose. The median contrast volume used was $80 \mathrm{~mL}$ (IQR $75-120 \mathrm{~mL}$ ). The median fluoroscopy time was $24.6 \mathrm{~min}-$ utes (IQR 22.9-33.2 minutes), and the median procedure time was 70.5 minutes (IQR 62-82 minutes). Finally, the median radiation dose was $38,978.5 \mathrm{mGy} / \mathrm{cm}^{2}$ (IQR 29,872-56,758 $\mathrm{mGy} / \mathrm{cm}^{2}$ ) (Table 3). 
TABLE 2. Procedural details of patients who underwent robot-assisted carotid artery stenting

\begin{tabular}{|c|c|c|c|c|c|c|}
\hline \multirow[b]{2}{*}{ Pt No. } & \multicolumn{6}{|c|}{ Variable } \\
\hline & Degree of Stenosis (\%) & Stenosis Diameter (mm) & Stent Type & Prestent Angioplasty & Balloon Type & Transradial Approach \\
\hline 1 & 95 & 1.8 & Precise Pro & Yes & Aviator Plus & Yes \\
\hline 2 & 51 & 3.04 & Precise Pro & Yes & Aviator & Yes \\
\hline 3 & 95 & 1.4 & Precise Pro & Yes & Aviator Plus & Yes \\
\hline 4 & 95 & 1.6 & Precise Pro & Yes & Aviator Plus & Yes \\
\hline 5 & 80 & 1.5 & Precise Pro & Yes & Aviator Plus & No \\
\hline 6 & 95 & 0.8 & Precise Pro & Yes & Aviator Plus & Yes \\
\hline 7 & 80 & 1.7 & Precise Pro & Yes & Aviator Plus & Yes \\
\hline 8 & 95 & 1.8 & Precise Pro & Yes & Aviator & No \\
\hline 9 & 99 & 0.6 & Precise Pro & Yes & Aviator Plus & Yes \\
\hline 10 & 80 & 1 & Precise Pro & Yes & Aviator & No \\
\hline 11 & 95 & 1 & Precise Pro & Yes & Aviator Plus & Yes \\
\hline 12 & 99 & 0.5 & Precise Pro & Yes & Aviator Plus & Yes \\
\hline 13 & 99 & 0.6 & Precise Pro & Yes & Aviator Plus & No \\
\hline 14 & 70 & 2.5 & Precise Pro & Yes & Aviator Plus & Yes \\
\hline Overall & $95(80-95)^{\star}$ & $1.45(0.8-1.8)^{*}$ & & $14(100) \dagger$ & & $10(71.4) \dagger$ \\
\hline
\end{tabular}

Pt = patient.

* Presented as the median (IQR).

† Presented as number of "yes" responses (\%).

In addition, we divided the 14 patients into 3 groups of 5,4 , and 5 patients (based on the procedure date) and looked at the temporal trend of these parameters with time. The mean duration for fluoroscopy and procedure time decreased from 32 minutes and 86 minutes, respectively, in group 1 to 21.9 minutes and 62 minutes, respectively, in group 3 , and the differences were statistically significant ( $p=0.002$ and $p=0.008$, respectively). Similarly, the total contrast volume and radiation dose decreased from group
1 to group 3; however, the trend did not reach statistical significance $(p=0.75$ and $p=0.13$, respectively) (Table 4 and Fig. 3A-D).

\section{Outcomes and Complications}

All procedures were conducted successfully, with no procedural complications. The median hospital length of stay was 4.5 days (IQR 3-8 days) and the median followup duration was 90 days (IQR 45-90 days). Nine patients

TABLE 3. Robot-assisted procedure parameters

\begin{tabular}{ccccc}
\hline & \multicolumn{4}{c}{ Variable } \\
\cline { 2 - 5 } Pt No. & Contrast Vol $(\mathrm{mL})$ & Fluoroscopy Time $($ mins) & Procedure Time $(\mathrm{mins})$ & Radiation Dose $\left(\mathrm{mGy} / \mathrm{cm}^{2}\right)$ \\
\hline 1 & 50 & 33.2 & 93 & 56,758 \\
\hline 2 & 75 & 34.9 & 92 & 29,872 \\
\hline 3 & 120 & 25.7 & 67 & 40,142 \\
\hline 4 & 100 & 24.6 & 72 & 73,765 \\
\hline 5 & 90 & 41.4 & 106 & 98,687 \\
\hline 6 & 150 & 22.9 & 82 & 25,078 \\
\hline 7 & 140 & 34.8 & 77 & 34,657 \\
\hline 8 & 80 & 25.2 & 75 & 66,193 \\
\hline 9 & 75 & 23.7 & 61 & 37,815 \\
\hline 10 & 120 & 23.3 & 67 & 33,571 \\
\hline 11 & 75 & 24.6 & 69 & 28,261 \\
\hline 12 & 75 & 19.3 & 53 & 48,845 \\
\hline 13 & 80 & 19.7 & 62 & 28,893 \\
\hline 14 & 75 & 22.8 & 59 & 41,718 \\
\hline Median total (IQR) & $80.150)$ & $24.6(22.9-33.2)$ & $70.5(62-82)$ & $38,978.5(29,872-56,758)$ \\
\hline patient. & & & &
\end{tabular}


Abbas et al.

TABLE 4. Trend for total contrast volume, fluoroscopy time, procedure time, and radiation dose across group 1 (patients 1-5), group 2 (patients 6-9), and group 3 (patients 10-14)

\begin{tabular}{ccccc}
\hline & \multicolumn{4}{c}{ Variable } \\
\cline { 2 - 5 } Group & Contrast Vol $(\mathrm{mL})$ & Fluoroscopy Time $(\mathrm{mins})$ & Procedural Time $(\mathrm{mins})$ & Radiation Dose $\left(\mathrm{mGy} / \mathrm{cm}^{2}\right)$ \\
\hline 1, mean & 87 & 32 & 86 & 59,845 \\
\hline 2, mean & 111.25 & 26.7 & 73.8 & 40,963 \\
\hline 3, mean & 85 & 21.9 & 62 & 36,258 \\
\hline p value & 0.7451 & $\mathbf{0 . 0 0 2}$ & $\mathbf{0 . 0 0 8}$ & 0.133 \\
\hline
\end{tabular}

Boldface type indicates statistical significance.

A

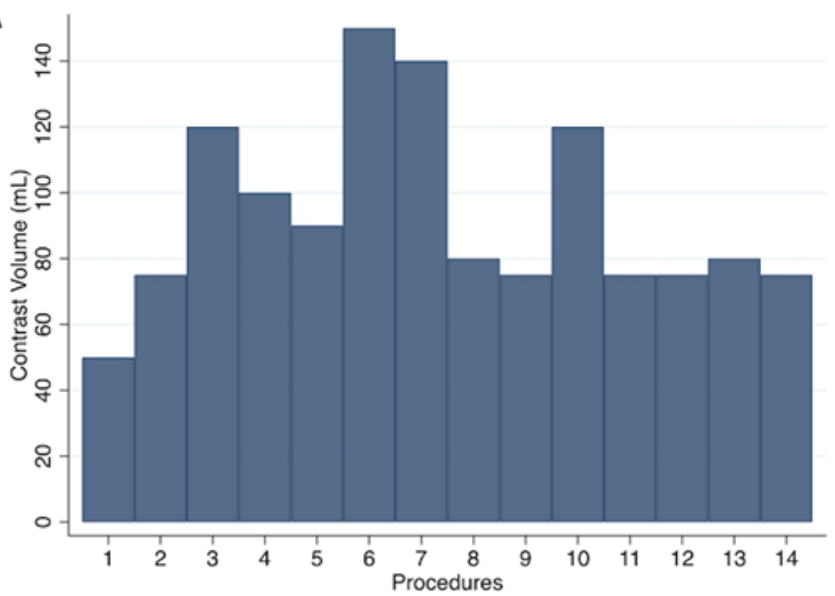

Test for trend, exact $\mathrm{p}$ value $=0.7451$ (100,000 Monte Carlo permutations) Group 1 mean $=87 \mathrm{~mL}$

Group 2 mean $=111.25 \mathrm{~mL}$

Group 3 mean $=85 \mathrm{~mL}$

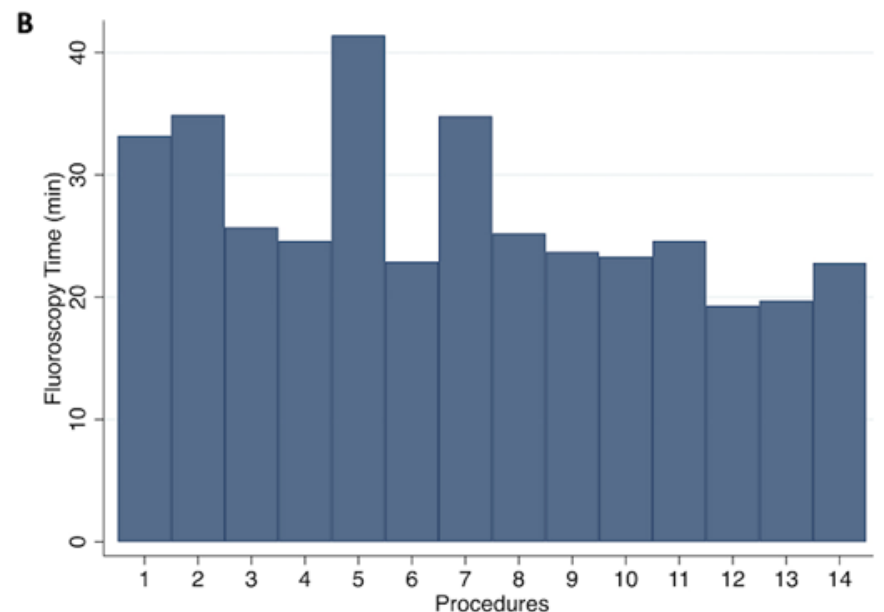

Test for trend, exact $\mathrm{p}$ value $=\mathbf{0 . 0 0 2}(100,000$ Monte Carlo permutations)

Group 1 mean $=32.0 \mathrm{~min}$

Group 2 mean $=26.7 \mathrm{~min}$

Group 3 mean $=21.9 \mathrm{~min}$

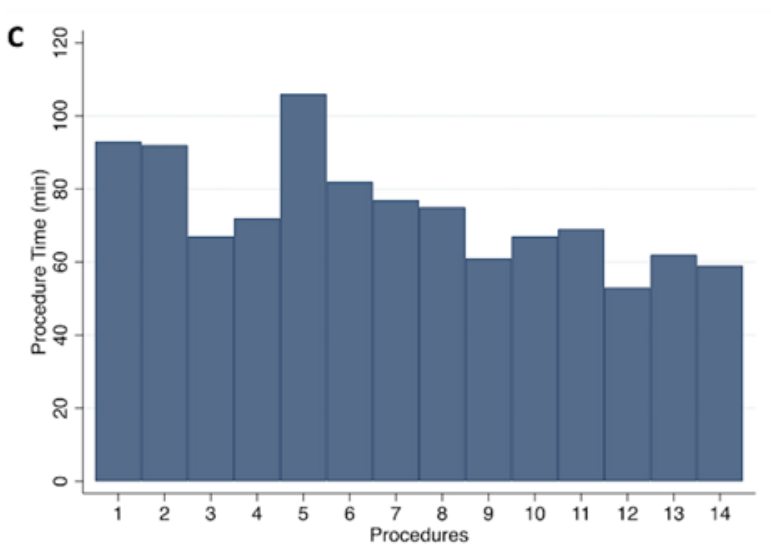

Test for trend, exact $\mathrm{p}$ value $=\mathbf{0 . 0 0 8}(100,000$ Monte Carlo permutations $)$ Group 1 mean $=86.0 \mathrm{~min}$

Group 2 mean $=73.8 \mathrm{~min}$ Group 3 mean $=62.0 \mathrm{~min}$

\section{。}

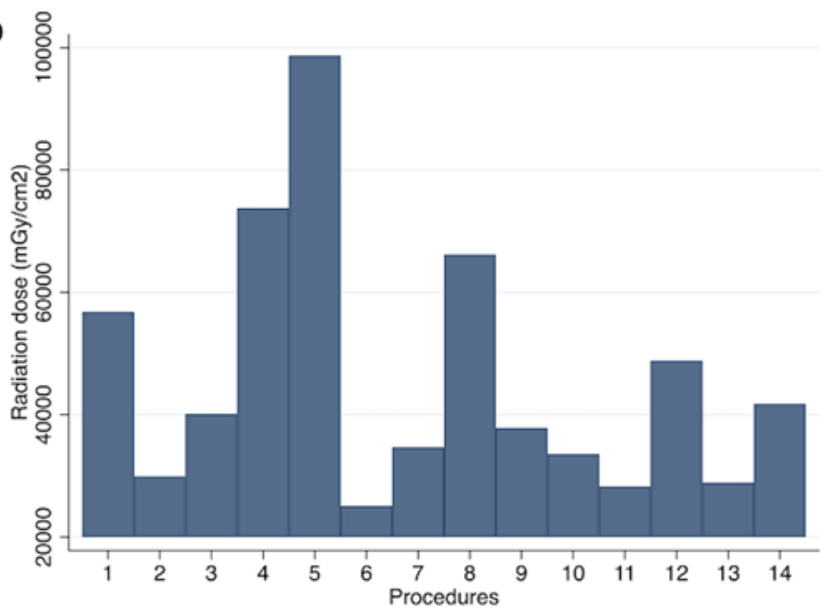

Test for trend, exact p value $=0.133$ (100,000 Monte Carlo permutations)

Group 1 mean $=59,845 \mathrm{mGy} / \mathrm{cm}^{2}$

Group 2 mean $=40,936 \mathrm{mGy} / \mathrm{cm}^{2}$

Group 3 mean $=36,258 \mathrm{mGy} / \mathrm{cm}^{2}$

FIG. 3. Bar graphs showing the trends for total contrast volume (A), fluoroscopy time $(\mathbf{B})$, total procedure time (C), and radiation dose (D) across procedures. 
TABLE 5. Outcomes of patients who underwent robot-assisted carotid artery stenting

\begin{tabular}{|c|c|c|c|c|c|c|c|}
\hline \multirow[b]{2}{*}{ Pt No. } & \multicolumn{7}{|c|}{ Variable } \\
\hline & Procedure Failure & Complications & LOS (days) & Mortality & mRS Score $0-2$ at 90 -Day FU & mRS Score $0-2$ at Final FU & FU Duration (days) \\
\hline 1 & No & No & 11 & No & NA & NA & NA \\
\hline 2 & No & No & 1 & No & Yes & Yes & 210 \\
\hline 3 & No & No & 12 & No & NA & NA & NA \\
\hline 4 & No & No & 19 & No & Yes & Yes & 90 \\
\hline 5 & No & No & 3 & No & Yes & Yes & 335 \\
\hline 6 & No & No & 5 & No & NA & NA & NA \\
\hline 7 & No & No & 3 & No & Yes & Yes & 90 \\
\hline 8 & No & No & 5 & No & NA & NA & NA \\
\hline 9 & No & No & 5 & No & Yes & Yes & 90 \\
\hline 10 & No & No & 1 & No & Yes & Yes & 60 \\
\hline 11 & No & No & 4 & Yes & NA & No & 4 \\
\hline 12 & No & No & 4 & No & Yes & Yes & 90 \\
\hline 13 & No & No & 1 & No & Yes & Yes & 45 \\
\hline 14 & No & No & 8 & No & Yes & Yes & 45 \\
\hline Overall & $0(0)^{*}$ & $0(0)^{*}$ & $4.5(3-8) \dagger$ & $1(7.1)^{*}$ & $9(100)^{*}$ & $9(90)^{*}$ & $90(45-90) \dagger$ \\
\hline
\end{tabular}

$\mathrm{FU}=$ follow-up; LOS = length of stay; NA = not applicable; $\mathrm{pt}=$ patient.

"Presented as number of "yes" responses (\%).

$\dagger$ Presented as the median (IQR).

(64\%) presented for the 90-day follow-up, and all 9 (100\%) had a favorable mRS score of 0 to 2 . At final follow-up, $90 \%$ of patients had a favorable mRS score. One patient (7\%) died 4 days after the procedure. The patient's death was unrelated to the procedure and was due to the severity of the initial presentation (Table 5).

\section{Discussion}

Neuroendovascular surgery is a continuously evolving specialty that aims to make use of novel techniques, tools, and technology in order to improve patient care. With the advancement of robotic engineering in surgery, along with the advantages it presents and the implications of its use on the future of healthcare, it is important for neurointerventionalists and postgraduate fellows to adapt to this technology.

Our study results show that robot-assisted carotid artery stenting is safe and feasible. All patients had successful procedures, and there were no technical or access-site complications. In addition, the procedures were able to be performed using both the transradial and transfemoral approaches. In a series of 10 patients who underwent both robot-assisted diagnostic cerebral angiograms as well as robot-assisted carotid artery stenting, Sajja et al. reported successful procedures in all patients with no complications encountered. ${ }^{12}$ Similarly, Nogueira et al. reported the feasibility of the CorPath GRX for carotid artery stenting in symptomatic disease. ${ }^{11}$ Moreover, multiple retrospective studies have shown that the transradial approach is as feasible as the transfemoral approach, with the exception of very few cases requiring transfemoral conversion due to tortuous anatomy or bovine arch configuration. ${ }^{4,11,12}$ This is very important, as the transradial approach has gained widespread adoption by interven- tionalists, with evidence showing increased patient preference as well..$^{13-15}$

As with any new technology or technique, there is a learning curve to overcome. We conducted a learning curve analysis and chose objective metrics including contrast volume, procedure and fluoroscopy times, and radiation dose. Our results showed a statistically significant decrease in fluoroscopy time between the first 5 robotassisted carotid artery stents (32 minutes) and the last 5 (21.9 minutes) $(\mathrm{p}=0.002)$. A similar, statistically significant decrease in procedure time (from 86 minutes to 62 minutes) was noted as well $(\mathrm{p}=0.008)$. These findings are supported by those of Weisz et al., which showed that only 3 robot-assisted cases were needed to achieve significant reductions in both procedure and fluoroscopy times $\left(p=0.008\right.$ and $p=0.003$, respectively). ${ }^{16}$ Similarly, Sajja et al. found that performance measures improved after 3 robot-assisted procedures, and Weinberg et al. reported improved procedure times with additional cases, without compromising patient safety.,12 In addition, an early comparison of manual versus robot-assisted carotid artery stenting showed that the mean procedure time was significantly longer in the robotic procedures (85.0 minutes vs 61.2 minutes, $\mathrm{p}=0.0231) .{ }^{4}$ However, with additional robotassisted cases, our current average procedure matches that of traditional manual cases.

Although not statistically significant, our study results also show a trend toward reduced radiation dose and contrast volume, signifying quicker procedures and further indicating a favorable learning curve that can be overcome with continued use. Our findings are in line with a study on the use of robotics for interventional cardiac procedures that reported operator prowess after 10 cases performed with the CorPath GRX. ${ }^{17}$ Our findings, and those in the 
literature, indicate a relatively short learning curve for robotic procedures compared with other new interventions. For example, performing 64 to 95 transradial cases are required for transfemoral interventionalists to overcome the learning curve for endovascular neurosurgery, and 39 to 50 cases for interventional cardiologists. ${ }^{16,18}$ Hence, this favorable learning curve for robot-assisted neurointerventions could encourage interventionalists to adopt this technology in their practice.

In addition to increased precision and accuracy, adopting robotic technology may reduce radiation exposure to the healthcare team by as much as $97 \%$, thereby decreasing the risk of malignancies, including left-sided brain tumors, breast cancer, and melanomas, the incidence of which usually increases with cumulative ionizing radiation..$^{9,19,20}$ Most importantly, robotic technology has the potential for executing procedures from remote locations. The CorPath GRX was successful in performing percutaneous coronary interventions from 20 and 100 miles away. ${ }^{5,6}$ Therefore, with specific neuroendovascular engineering and software modifications ${ }^{21}$ a short learning curve, and eventual FDA approval for intracranial procedures, robotic systems bear a great potential to provide life-saving procedures, including mechanical thrombectomies and ruptured aneurysm embolizations, to patients in rural areas who are without immediate access to cerebrovascular centers.

Depending on regional or institutional variability, the average cost of the CorPath GRX robot system is $\$ 500,000 .{ }^{22}$ Furthermore, the disposable cassette costs an additional $\$ 559$ for each procedure. This may deter some hospitals and interventionalists, especially small hospital systems in rural areas, from adopting this technology. However, patient safety and the lower cumulative radiation exposure of physicians must be taken into consideration. Also, as technology is continuously improving, there is potential to establish neuroendovascular networks capable of performing emergency procedures remotely. Therefore, the seemingly expensive cost may be justified, as it may not only save more lives but also reduce costs long-term, given the healthcare expenditures associated with stroke morbidity. ${ }^{23}$

Due to the numerous advantages that the CorPath GRX robot system provides, it is important that its use is incorporated as a component of neuroendovascular fellowship training whenever possible. This can include formal, proprietary in-service training as well as continuous supervision by faculty to ensure that operator proficiency is achieved.

\section{Limitations}

This study is limited by its retrospective design, lack of randomization, lack of long-term patient follow-up, lack of objective criteria in choosing the robotic technique, and small sample size. Also, the data are from a single center in the US and, therefore, the generalizability of the results is limited. However, learning curve analysis was done with objective metrics. Our findings show that the learning curve for robot-assisted neuroendovascular procedures can be overcome. However, future prospective clinical trials are needed to further elucidate their safety, efficacy, and consistency, and to determine the subsequent direction.

\section{Conclusions}

Our study results show that robot-assisted carotid artery stenting is safe and efficacious, and that the learning curve can be overcome with relatively few cases at a highvolume cerebrovascular center. However, the full potential of robotics in neuroendovascular surgery has yet to be achieved and future clinical studies are needed to consolidate its role in the future of neurological surgery.

\section{References}

1. Maor E, Eleid MF, Gulati R, Lerman A, Sandhu GS. Current and future use of robotic devices to perform percutaneous coronary interventions: a review. J Am Heart Assoc. 2017; 6(7):e006239.

2. Mendes Pereira V, Cancelliere NM, Nicholson P, et al. Firstin-human, robotic-assisted neuroendovascular intervention. $J$ Neurointerv Surg. 2020;12(4):338-340.

3. Vuong SM, Carroll CP, Tackla RD, Jeong WJ, Ringer AJ. Application of emerging technologies to improve access to ischemic stroke care. Neurosurg Focus. 2017;42(4):E8.

4. Weinberg JH, Sweid A, Sajja K, et al. Comparison of robotic-assisted carotid stenting and manual carotid stenting through the transradial approach. J Neurosurg. 2021;135(1): 21-28.

5. Patel TM, Shah SC, Pancholy SB. Long distance telerobotic-assisted percutaneous coronary intervention: a report of first-in-human experience. EClinicalMedicine. 2019;14: 53-58.

6. Madder RD, VanOosterhout S, Mulder A, et al. Feasibility of robotic telestenting over long geographic distances: a preclinical ex vivo and in vivo study. EuroIntervention. 2019; 15(6):e510-e512.

7. De Benedictis A, Trezza A, Carai A, et al. Robot-assisted procedures in pediatric neurosurgery. Neurosurg Focus. 2017;42(5):E7.

8. Ghasem A, Sharma A, Greif DN, Alam M, Maaieh MA. The arrival of robotics in spine surgery: a review of the literature. Spine (Phila Pa 1976). 2018;43(23):1670-1677.

9. Granada JF, Delgado JA, Uribe MP, et al. First-in-human evaluation of a novel robotic-assisted coronary angioplasty system. JACC Cardiovasc Interv. 2011;4(4):460-465.

10. Weisz G, Metzger DC, Caputo RP, et al. Safety and feasibility of robotic percutaneous coronary intervention: PRECISE (Percutaneous Robotically-Enhanced Coronary Intervention) Study. J Am Coll Cardiol. 2013;61(15):1596-1600.

11. Nogueira RG, Sachdeva R, Al-Bayati AR, Mohammaden MH, Frankel MR, Haussen DC. Robotic assisted carotid artery stenting for the treatment of symptomatic carotid disease: technical feasibility and preliminary results. J Neurointerv Surg. 2020;12(4):341-344.

12. Sajja KC, Sweid A, Al Saiegh F, et al. Endovascular robotic: feasibility and proof of principle for diagnostic cerebral angiography and carotid artery stenting. J Neurointerv Surg. 2020;12(4):345-349.

13. Khanna O, Sweid A, Mouchtouris N, et al. Radial artery catheterization for neuroendovascular procedures. Stroke. 2019;50(9):2587-2590.

14. Chen SH, Snelling BM, Sur S, et al. Transradial versus transfemoral access for anterior circulation mechanical thrombectomy: comparison of technical and clinical outcomes. $J$ Neurointerv Surg. 2019;11(9):874-878.

15. Kok MM, Weernink MGM, von Birgelen C, Fens A, van der Heijden LC, van Til JA. Patient preference for radial versus 
femoral vascular access for elective coronary procedures: the PREVAS study. Catheter Cardiovasc Interv. 2018;91(1): 17-24.

16. Weisz G, Smilowitz NR, Metzger DC, et al. The association between experience and proficiency with robotic-enhanced coronary intervention-insights from the PRECISE multicenter study. Acute Card Care. 2014;16(2):37-40.

17. Ragosta M, Singh KP. Robotic-assisted percutaneous coronary intervention: rationale, implementation, case selection and limitations of current technology. J Clin Med. 2018;7(2): E23.

18. Al Saiegh F, Sweid A, Chalouhi N, et al. Comparison of transradial vs transfemoral access in neurovascular fellowship training: overcoming the learning curve. Oper Neurosurg (Hagerstown). 2021;21(1):E3-E7.

19. Roguin A, Goldstein J, Bar O, Goldstein JA. Brain and neck tumors among physicians performing interventional procedures. Am J Cardiol. 2013;111(9):1368-1372.

20. Rajaraman P, Doody MM, Yu CL, et al. Cancer risks in U.S. radiologic technologists working with fluoroscopically guided interventional procedures, 1994-2008. AJR Am J Roentgenol. 2016;206(5):1101- 1109.

21. Britz GW, Panesar SS, Falb P, Tomas J, Desai V, Lumsden A. Neuroendovascular-specific engineering modifications to the CorPath GRX Robotic System. J Neurosurg. 2020;133(6): 1830-1836.

22. Cairns E. \$1bn Corindus deal gets Healthineers off the acquisition blocks. Evaluate Vantage. Accessed November 16, 2021. https://www.evaluate.com/vantage/articles/news/ deals/1bn-corindus-deal-gets-healthineers-acquisition-blocks
23. Abdo RR, Abboud HM, Salameh PG, Jomaa NA, Rizk RG, Hosseini HH. Direct medical cost of hospitalization for acute stroke in Lebanon: a prospective incidence-based multicenter cost-of-illness study. Inquiry. 2018;55:46958018792975.

\section{Disclosures}

Dr. Jabbour is a consultant for Medtronic and MicroVention. Dr. Tjoumakaris and Dr. Gooch are consultants for Stryker. Dr. Tjoumakaris is a consultant for Medtronic and MicroVention.

\section{Author Contributions}

Conception and design: Jabbour, Abbas, Al Saiegh. Acquisition of data: Abbas, Al Saiegh, El Naamani, Sioutas, Weinberg. Analysis and interpretation of data: Jabbour, Abbas, Chen. Drafting the article: Abbas. Critically revising the article: Jabbour, Abbas, Al Saiegh, El Naamani, Tjoumakaris, Gooch, Herial, Rosenwasser. Reviewed submitted version of manuscript: all authors. Approved the final version of the manuscript on behalf of all authors: Jabbour. Statistical analysis: Chen, Velagapudi. Study supervision: Jabbour.

\section{Correspondence}

Pascal Jabbour: Thomas Jefferson University Hospital, Philadelphia, PA.pascal.jabbour@jefferson.edu. 